\begin{tabular}{|c|c|}
\hline Title & On embedded bifurcation structure in some discretized vector fields \\
\hline Author(s) & Kang, Hunseok; Tsuda, Ichiro \\
\hline Citation & $\begin{array}{l}\text { Chaos: An Interdisciplinary Journal of Nonlinear Science, 19(3), } 033132 \\
\text { https://doi.org/10.1063/1.3212934 }\end{array}$ \\
\hline Issue Date & 2009-09 \\
\hline Doc URL & http:/hdl.handle.net/2115/39276 \\
\hline Rights & $\begin{array}{l}\text { Copyright } 2009 \text { A merican Institute of Physics. This article may be downloaded for personal use only. A ny other use } \\
\text { requires prior permission of the author and the A merican Institute of Phy sics. The following article appeared in Chaos, } \\
19,033132,2009 \text { and may be found at } h \text { tttp://dx.doi.org/10.1063/1.3212934 }\end{array}$ \\
\hline Type & article \\
\hline File Information & CHAOEH193033132_1.pdf \\
\hline
\end{tabular}

Instructions for use 


\title{
On embedded bifurcation structure in some discretized vector fields
}

\author{
Hunseok Kang $^{\text {a) }}$ and Ichiro Tsuda ${ }^{\text {b) }}$ \\ Research Institute for Electronic Science, Hokkaido University, Kita-20, Nishi-10, Kita-ku, \\ Sapporo 001-0020, Japan and Department of Mathematics, Graduate School of Science, \\ Hokkaido University, Kita-10, Nishi-8, Kita-ku, Sapporo 060-0810, Japan
}

(Received 2 March 2009; accepted 6 August 2009; published online 9 September 2009)

In this paper, we study a dynamic structure of discretized vector fields obtained from the Brusselator, which is described by two-dimensional ordinary differential equations (ODEs). We found that a bifurcation structure of the logistic map is embedded in the discretized vector field. The embedded bifurcation structure was unraveled by the dynamical orbits that eventually converge to a fixed point. We provide a detailed mathematical analysis to explain this phenomenon and relate it to the solution of the original ODEs. (C) 2009 American Institute of Physics. [DOI: 10.1063/1.3212934]

A qualitative change in the dynamics, which occurs as a system parameter varies, is called a bifurcation. A bifurcation diagram is a well-known utility in the dynamical systems to observe the qualitative changes such as the emergence and vanishment of periodic orbits and the alteration of their stabilities. In this paper, we shall study some dynamical orbits of a discretized vector field in which a bifurcation structure is embedded. Strange as it may sound, the embedded bifurcation structure has no relation to such qualitative changes representing bifurcations. We observe that some dynamical orbits of a twodimensional map exhibit bifurcation structure in a phase space and eventually converge to their equilibrium. A crucial clue to understand the emergence of the embedded bifurcation structures is given in this paper, that is, the bifurcations of a random logistic map. In addition, the convergence of the dynamical orbits is guaranteed by the existence of an arbitrarily small bounded trapping region containing the equilibrium.

\section{INTRODUCTION}

In 1968, Prigogine and Lefever proposed a hypothetical model of chemical reactions showing oscillations, called Brusselator. This model is one of the simplest chemical models exhibiting a pattern forming instability called Turing instability. In the absence of diffusion, only accounting for the reaction kinetics, we have a system of ordinary differential equations (ODEs) in dimensionless form

$$
\frac{d x}{d t}=A-(1+B) x+x^{2} y, \quad \frac{d y}{d t}=B x-x^{2} y,
$$

where $A$ and $B$ are positive constants, which govern the pattern selection in the model by defining the reaction kinetics. For detailed explanations of this model see Ref. 1.

We consider Euler's discretization of the ODEs for Brusselator in Eq. (1). Euler's discretization scheme for Eq. (1) is as follows. With a fixed time discretization step $\Delta t$,

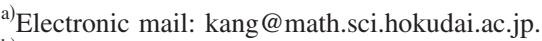

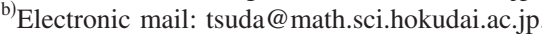

$$
\begin{aligned}
& x_{n+1}=x_{n}+\Delta t\left(A-(1+B) x_{n}+x_{n}^{2} y_{n}\right), \\
& y_{n+1}=y_{n}+\Delta t\left(B x_{n}-x_{n}^{2} y_{n}\right) .
\end{aligned}
$$

Then we obtain a nonlinear two-dimensional dynamical system $f: \mathbb{R}^{2} \rightarrow \mathbb{R}^{2}$ given by $f(x, y)=\left(f_{1}(x, y), f_{2}(x, y)\right)$ such that

$$
\begin{aligned}
& f_{1}(x, y)=a+(1-\gamma-b) x+\gamma x^{2} y, \\
& f_{2}(x, y)=y+b x-\gamma x^{2} y,
\end{aligned}
$$

where the parameters are

$$
a=A \Delta t, \quad b=B \Delta t, \quad \text { and } \gamma=\Delta t .
$$

We here call map $f$ a direction field, in contrast to a vector field of the original Brusselator.

In this paper, we present a study of the dynamics of map $f$ in the direction field in which a bifurcation structure is embedded. The bifurcation structure emerges out of some dynamical orbits of $f$, and the structure eventually disappears while the orbit approaches to the equilibrium. We show that the embedded bifurcation structure is based on the tangent bifurcation and the period-doubling bifurcation, which occur in the dynamics of a type of logistic map. We also investigate the limiting behavior of the dynamical orbits displaying the bifurcation structures. Depending on the stabilities of the fixed point(s), the orbits have the possibility to converge to a fixed point, form a limit cycle around the fixed point, or escape to infinity. We find a sufficient condition of parameters for the existence of arbitrarily small bounded trapping regions containing the fixed point. This implies that the orbits are confined in an arbitrarily small trapping region and eventually converge to the fixed point.

Discretization is concerned with the transformation of continuous differential equations into discrete difference equations, suitable for numerical computing. In general, it is unfavorable that the analytic properties of the solutions in the original system are obtained from the study of its discretized map because various types of errors may occur and be accumulated at each iteration in its trajectories and, hence, the stroboscopic motion in an orbit of the discretized map $f$ may deviate from the right trajectory. In this paper, we shall dis- 
cuss in detail how the dynamical properties obtained from the discretization are applied to the flows of the solutions in the original system in avoiding the risks mentioned above.

\section{PRELIMINARIES}

In this section, we arrange the notations and assumptions that are used in this paper. Also, a linear analysis about the stability of map $f$ is carried out. We rewrite the twodimensional dynamical system $f=\left(f_{1}, f_{2}\right)$ in the following form:

$$
\begin{aligned}
& x_{n+1}=f_{1}\left(x_{n}, y_{n}\right)=a+(1-\gamma-b) x_{n}+\gamma x_{n}^{2} y_{n}, \\
& y_{n+1}=f_{2}\left(x_{n}, y_{n}\right)=y_{n}+b x_{n}-\gamma x_{n}^{2} y_{n},
\end{aligned}
$$

where $f_{1}$ and $f_{2}$ are defined in Eqs. (2) and (3), respectively.

We clarify certain conditions of the parameters to be assumed in this paper. As seen in Eq. (4), the parameter $\gamma$ is the discretization step $\Delta t$ for Eq. (1), and $a$ and $b$ are mul- tiples of the discretization step $\Delta t$. Thus, without imposing much of the physical constraints to the system, it is plausible to assume that

(1) $\gamma$ is sufficiently small and

(2) $a / \gamma=O(1)$ and $b / \gamma=O(1)$,

where $O(1)$ indicates that $a / \gamma$ and $b / \gamma$ are of the order of 1 .

For all positive values of $a, b$, and $\gamma$, there is only one fixed point of $f$

$$
\mathbf{p}^{*}:=\left(x^{*}, y^{*}\right)=\left(\frac{a}{\gamma}, \frac{b}{a}\right) .
$$

We use the transformation matrix $D f_{\mathbf{p}}$ to analyze the linear stability of the system. For a point $\mathbf{p}=(x, y) \in \mathbb{R}^{2}$,

$$
D f_{\mathbf{p}}=\left[\begin{array}{rr}
1-\gamma-b+2 \gamma x y & \gamma x^{2} \\
b-2 \gamma x y & 1-\gamma x^{2}
\end{array}\right] .
$$

The eigenvalues $\lambda_{\mathbf{p}}^{(i)}(i=1,2)$ of $D f_{\mathbf{p}}$ are

$$
\lambda_{\mathbf{p}}^{(i)}=1-\frac{\gamma+b-2 \gamma x y+\gamma x^{2} \pm \sqrt{\left(\gamma+b-2 \gamma x y+\gamma x^{2}\right)^{2}-4 \gamma^{2} x^{2}}}{2} .
$$

Especially, the eigenvalues at the fixed point $\mathbf{p}^{*}$ are

$$
\lambda_{\mathbf{p}^{*}}^{(i)}=1-\frac{\gamma-b+a^{2} / \gamma \pm \sqrt{\left(\gamma-b+a^{2} / \gamma\right)^{2}-4 a^{2}}}{2} .
$$

Under the assumptions that $a$ and $\gamma$ are sufficiently small and $a / \gamma=O(1)$, we have

$$
\lambda_{\mathbf{p}^{*}}^{(1)}=1+\epsilon_{1}, \quad \lambda_{\mathbf{p}^{*}}^{(2)}=1+b+\epsilon_{2},
$$

where $\epsilon_{i} \rightarrow 0(i=1,2)$ as $a$ and $\gamma \rightarrow 0$. In Refs. 2 and 3 there are more detailed discussions concerning the stabilities of the fixed point.

Referring to Eqs. (7) and (8), it is notable that the values of parameters $a, b$, and $\gamma$ can be rescaled while holding the same qualitative dynamical properties concerning the stability of the system topologically. More precisely, when $\Delta t$ is rescaled by a small scaling factor $\kappa<1$, since $a, b$, and $\gamma$ are multiples of $\Delta t$, the fixed point is unchanged, that is, $\mathbf{p}^{*}$ $=(a / \gamma, b / a)$, and the eigenvalues in Eq. (7) at $\mathbf{p}=(x, y)$ are replaced by

$$
\begin{aligned}
\lambda_{\mathbf{p}}^{(i)}= & 1-\frac{\kappa}{2}\left(\gamma+b-2 \gamma x y+\gamma x^{2}\right. \\
& \left. \pm \sqrt{\left(\gamma+b-2 \gamma x y+\gamma x^{2}\right)^{2}-4 \gamma^{2} x^{2}}\right)
\end{aligned}
$$

We define two sets of curves in the plane as follows:

$$
\begin{aligned}
& N_{1}=\left\{(x, y): f_{1}(x, y)-x=a-(\gamma+b) x+\gamma x^{2} y=0\right\}, \\
& N_{2}=\left\{(x, y): f_{2}(x, y)-y=b x-\gamma x^{2} y=0\right\} .
\end{aligned}
$$

Clearly, $N_{1} \cap N_{2}$ determines the fixed point $\mathbf{p}^{*}$. Moreover, $N_{1}$ and $N_{2}$ play the same role as the nullclines of the ODEs for the Brusselator given in Eq. (1). The curves in $N_{1}$ and $N_{2}$ indicate the boundaries beyond which a point in the plane moves right or left by $f_{1}$ and moves upward or downward by $f_{2}$. In fact, the nullclines of the Brusselator are exactly the same as $N_{1}$ and $N_{2}$ defined in Eq. (9). Therefore, without confusion, we name $N_{1}$ and $N_{2}$ nullclines for $f_{1}$ and $f_{2}$, respectively.

\section{BIFURCATION STRUCTURES}

We look into the mechanism of how the orbits of $f$ generate the bifurcation structure. The creation of the dynamical orbits in which a bifurcation structure is embedded is involved in the procedure of the bifurcations in a random logistic map. We shall give a detailed mathematical justification as to why the bifurcation structures are embedded in the direction field $f$ defined in Eqs. (2) and (3) and compute the exact location of the bifurcation structures.

Regarding the variable $y$ in Eq. (2) as a parameter, we obtain a one-dimensional map $g_{y}(x)=f_{1}(x, y)$, where

$$
g_{y}(x)=\gamma y x^{2}+(1-\gamma-b) x+a .
$$

Then map $g_{y}$ can be viewed as a random logistic map. We compare the following two dynamical structures (see Fig. 1):

(1) a bifurcation diagram of the one-dimensional map $g_{y}$ where the bifurcation parameter $y$ increases from a proper negative value and

(2) a dynamical orbit $\left\{f^{n}\left(x_{0}, y_{0}\right)\right\}_{n=0}^{\infty}$, where $\left|x_{0}\right|$ is sufficiently small and $y_{0}$ is a well-chosen negative value. 

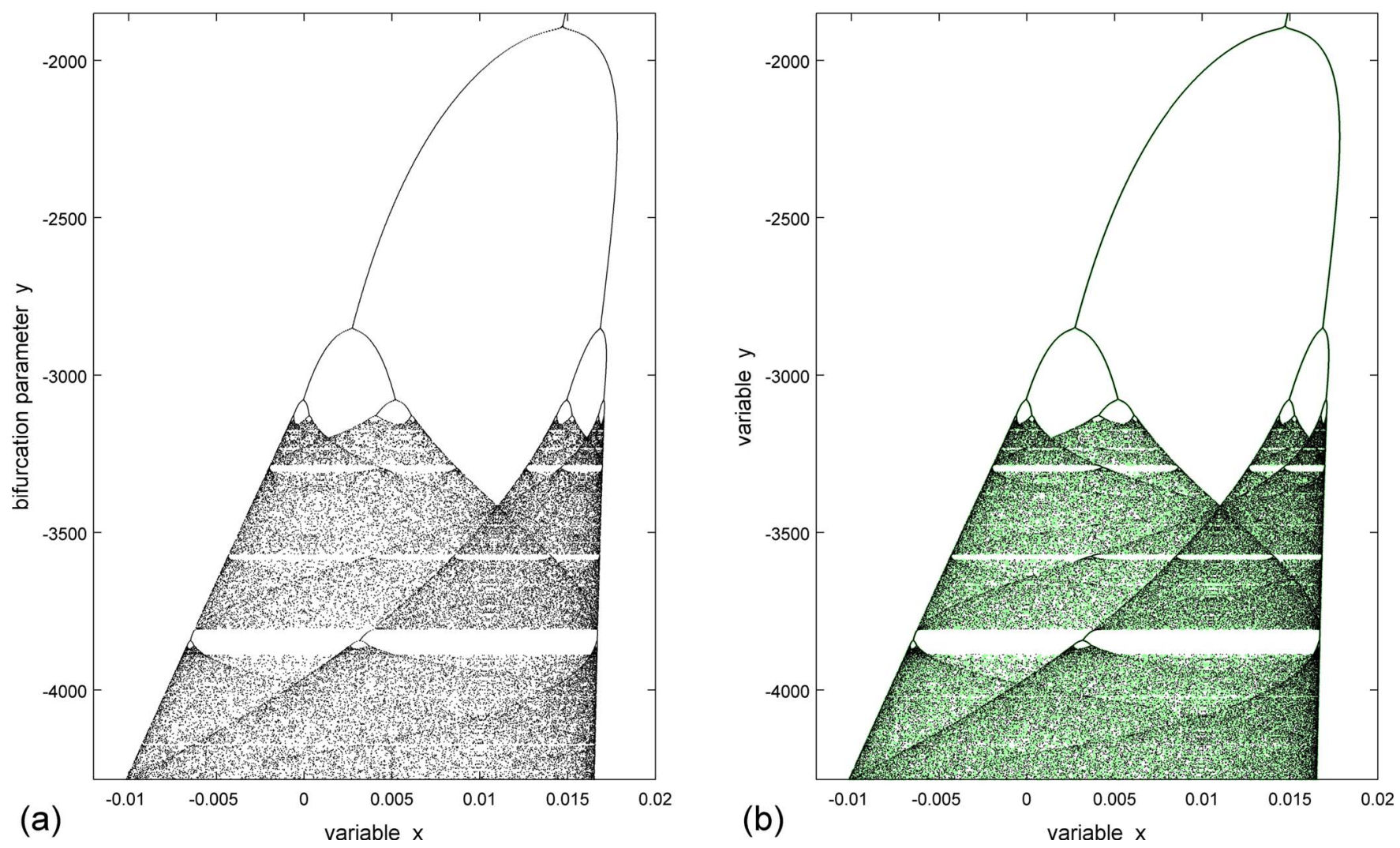

FIG. 1. (Color online) Comparison of a dynamical orbit of $f$ to a bifurcation diagram of $g_{y}$ at the same parameter values of $a=0.015, b=0.01$, and $\gamma$ $=0.035$. (a) The bifurcation diagram of a random logistic map. The bifurcation parameter $y$ of $g_{y}(x)$ defined in Eq. (10) varies from -4284 to -1850 . (b) A dynamical orbit of map $f$. This orbit originates in the point $(0,-4284)$, and about 160000 iterations are displayed in the background of the bifurcation diagram in (a).

In general, the bifurcation of a logistic map occurs only when there exists an invariant interval under the map. Thus, our current goals are (1) to determine the largest upper bound and the smallest lower bound of the parameter $y$ in Eq. (10) so that for all $y$ between the two bounds there exists an invariant interval under map $g_{y}$ and (2) to find the maximum invariant interval $E_{y}$ under map $g_{y}$, i.e., $g_{y}\left(E_{y}\right) \subset E_{y}$. Here, we set $y^{*}$ as the largest upper bound of the parameter $y$. To find the smallest lower bound of $y$ and to specify the maximum invariant interval $E_{y}$ for each $y$, we consider the following cases where $y<0, y=0$, and $0<y \leq y^{*}$ in order.

Case $1[y<0]$. The equation $g_{y}(x)=x$ yields two fixed points of $g_{y}, r_{y}^{(1)}$ and $r_{y}^{(2)}$, with $r_{y}^{(1)}<0<r_{y}^{(2)}$,

$$
\begin{aligned}
& r_{y}^{(1)}=\frac{(\gamma+b)+\sqrt{(\gamma+b)^{2}-4 a \gamma y}}{2 \gamma y}, \\
& r_{y}^{(2)}=\frac{(\gamma+b)-\sqrt{(\gamma+b)^{2}-4 a \gamma y}}{2 \gamma y} .
\end{aligned}
$$

The maximum value $m_{y}$ of $g_{y}$ is

$$
m_{y}=a-\frac{(1-\gamma-b)^{2}}{4 \gamma y} \text { at } x=-\frac{1-\gamma-b}{2 \gamma y} .
$$

Let $\alpha_{y}$ and $\beta_{y}$ be the endpoints of $E_{y}$. In order for the interval $E_{y}$ to be invariant under map $g_{y}$, we need the following:
(1) two endpoints $\alpha_{y}$ and $\beta_{y}$ are two roots of the equation $g_{y}(x)=r_{y}^{(1)}$ and

(2) the rate of normalization of $g_{y}\left(E_{y}\right)$ by $E_{y}$ is less than or equal to 1

[see Fig. 2(a)]. The equation $g_{y}(x)=\gamma y x^{2}+(1-\gamma-b) x+a$ $=r_{y}^{(1)}$ has two roots, and these roots must be $\alpha_{y}$ and $\beta_{y}$,

$$
\begin{aligned}
& \alpha_{y}=\frac{(\gamma+b)+\sqrt{(\gamma+b)^{2}-4 a \gamma y}}{2 \gamma y}, \\
& \beta_{y}=\frac{-2+\gamma+b-\sqrt{(\gamma+b)^{2}-4 a \gamma y}}{2 \gamma y} .
\end{aligned}
$$

The normalization rate $\varphi(y)$ of $g_{y}\left(E_{y}\right)$ by $E_{y}$ is

$$
\varphi(y)=\frac{m_{y}-\alpha_{y}}{\beta_{y}-\alpha_{y}}=\frac{1+\sqrt{(\gamma+b)^{2}-4 a \gamma y}}{4} .
$$

From the inequality $\varphi(y) \leq 1$, we obtain

$$
\varphi(y) \leq 1 \Rightarrow y \geq-\frac{9-(\gamma+b)^{2}}{4 a \gamma} .
$$

Therefore, for all $y \in\left[y_{(\mathrm{ini})}, 0\right)$, the interval $E_{y}=\left[\alpha_{y}, \beta_{y}\right]$ is invariant under $g_{y}$, where

$$
y_{\text {(ini) }}:=-\frac{9-(\gamma+b)^{2}}{4 a \gamma} .
$$




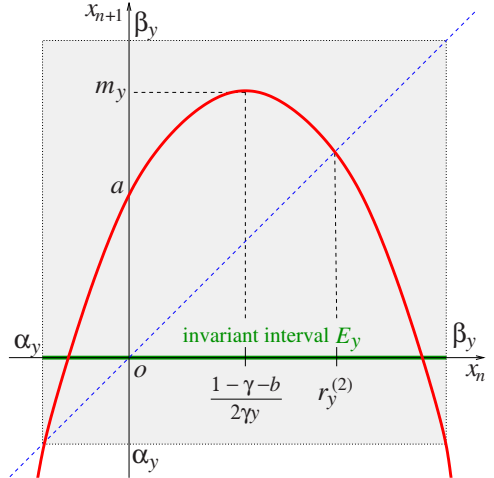

(a) Case $1[y<0]$.

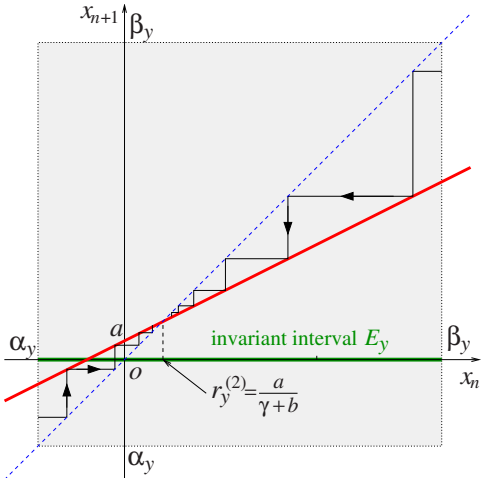

(b) Case $2[y=0]$.

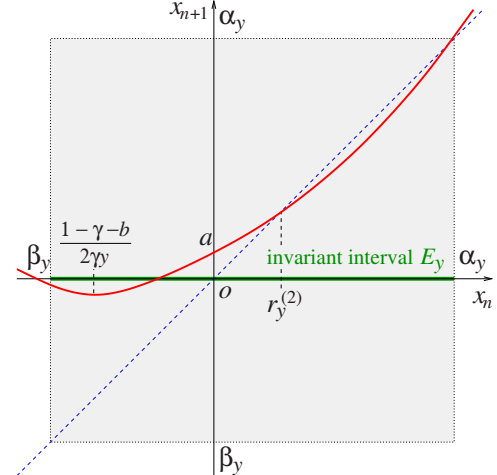

(c) Case $3\left[0<y \leq y^{*}\right]$.

FIG. 2. (Color online) The graphs of $g_{y}$ (thick curves) and the invariant intervals $E_{y}$ of $g_{y}$ (line segments on the $x_{n}$-axis). (a) $E_{y}=\left[\alpha_{y}, \beta_{y}\right]$ when $y_{\text {(ini) }} \leq y$ $<0$. (b) $E_{y}=\mathbb{R}$ when $y=0$. (c) $E_{y}=\left[\beta_{y}, \alpha_{y}\right]$ when $0<y \leq y^{*}$. The fixed point $r_{y}^{(2)}$ of $g_{y}$ is a sink as shown in (b) and (c).

Case $2[y=0]$. From the equation

$$
g_{y}(x)=(1-\gamma-b) x+a,
$$

we have only one fixed point $a /(\gamma+b)$ of $g_{y}$. For all $x \in \mathbb{R}$,

$$
\left|g_{y}^{\prime}(x)\right|=|1-\gamma-b|<1,
$$

so the fixed point $a /(\gamma+b)$ is a sink, and every interval containing the fixed point is invariant under $g_{y}$. Thus, we set $E_{y}=\mathrm{R}$ [see Fig. 2(b)].

Case $3\left[0<y \leq y^{*}\right]$. The fixed points of $g_{y}$ are still $r_{y}^{(1)}$ and $r_{y}^{(2)}$, given in Eq. (11). In this case, since the graph of $g_{y}$ is convex from above, the inequalities

$$
0<r_{y}^{(2)}<r_{y}^{(1)} \text { and } \beta_{y}<\alpha_{y},
$$

hold, and the value $m_{y}$ in Eq. (12) is the minimum of $g_{y}$. Similar to Case 1, one can show that $\varphi(y) \leq 1$ for all $y$ $\in\left(0, y^{*}\right]$. Thus, $E_{y}=\left[\beta_{y}, \alpha_{y}\right]$ is the maximum interval invariant under $g_{y}$ [see Fig. 2(c)].

Consequently, for each $y \in\left[y_{(\text {ini) }}, y^{*}\right]$, there exists the maximum interval $E_{y}$ invariant under map $g_{y}$. We slightly modify the notation of $r_{y}^{(2)}$, defined in Eq. (11), as follows:

$$
r_{y}^{(2)}=\frac{2 a}{(\gamma+b)+\sqrt{(\gamma+b)^{2}-4 a \gamma y}} .
$$

Since $r_{y}^{(2)}=a /(\gamma+b)$ holds at $y=0, r_{y}^{(2)} \in E_{y}$, and $r_{y}^{(2)}$ can be regarded as a fixed point of the map $g_{y}$ for all $y \in\left[y_{(\mathrm{ini})}, y^{*}\right]$.

We inspect the stability of $g_{y}$ at the fixed points $r_{y}^{(1)}$ and $r_{y}^{(2)}$. For all $y \in\left[y_{(\text {ini) }}, 0\right) \cup\left(0, y^{*}\right]$, we have

$$
\left.\frac{d g_{y}}{d x}\right|_{x=r_{y}^{(1)}}=1+\sqrt{(\gamma+b)^{2}-4 a \gamma y}>1,
$$

and hence, $r_{y}^{(1)}$ is always a source of $g_{y}$. However, the stability of $r_{y}^{(2)}$ changes at a value $y_{(\text {bif })} \in\left[y_{(\text {ini) }}, 0\right]$. For $y=y_{(\text {ini) }}$, $\beta_{y}=m_{y}$, so $r_{y}^{(2)}$ is a source [see Fig. 2(a)]. As $y$ increases from $y_{\text {(ini) }}$ to 0 ,

(1) the maximum value $m_{y}$, given in Eq. (12), monotone increases and

(2) the length of the interval $E_{y}$, which is $\left|\beta_{y}-\alpha_{y}\right|$, monotone increases.
For $y_{(\text {ini) }} \leq y<0$, the derivative of the normalization rate $\varphi(y)$ is

$$
\varphi^{\prime}(y)=-\frac{a \gamma}{2 \sqrt{(\gamma+b)^{2}-4 a \gamma y}}<0 .
$$

Thus, the increasing rate of $m_{y}$ is less than that of the length of $E_{y}$, and hence, there exists a constant $y_{(\text {bif })}<0$ such that $r_{y}^{(2)}$ is a sink for $y \in\left(y_{(\mathrm{bif})}, 0\right)$, while it is a source for $y$ $\in\left[y_{\text {(ini) }}, y_{\text {(bif) }}\right)$. At $y=y_{\text {(bif) }}$ the last bifurcation of $g_{y}$ occurs, and its exact value is obtained from the following equality:

$$
\left.\frac{d g_{y}}{d x}\right|_{x=r_{y}^{(2)}}=1-\sqrt{(\gamma+b)^{2}-4 a \gamma y}=-1 .
$$

Therefore, we have

$$
y_{\text {(bif) }}=-\frac{4-(\gamma+b)^{2}}{4 a \gamma} .
$$

Using the same argument as above, one can show that $r_{y}^{(2)}$ is also a sink for all $y \in\left(0, y^{*}\right]$.

We define two sets of curves as follows:

$$
\begin{aligned}
& R_{1}=\left\{\left(r_{y}^{(1)}, y\right) \mid y_{(\mathrm{ini})} \leq y<0,0<y \leq y^{*}\right\}, \\
& R_{2}=\left\{\left(r_{y}^{(2)}, y\right) \mid y_{\text {(ini) }} \leq y \leq y^{*}\right\} .
\end{aligned}
$$

Clearly, $R_{i} \subset N_{1}$ for $i=1,2$.

Remark 1: The random logistic map $g_{y}$ possesses the period-doubling bifurcation and the tangent bifurcation which appear around $R_{1}$ and $R_{2}$. Specifically, we have the following.

(1) When $y_{(\mathrm{bif})}<y<y^{*}$, while $r_{y}^{(1)}(y \neq 0)$ is a source of $g_{y}$, $r_{y}^{(2)}$ is a sink of $g_{y}$. Thus, all attracting orbits of $g_{y} a p-$ proach the curve $R_{2}$, and there exists the tangent bifurcation.

(2) When $y_{(\mathrm{ini})}<y<y_{(\mathrm{bif})}$, both $r_{y}^{(1)}$ and $r_{y}^{(2)}$ are sources. Thus, almost all orbits of $g_{y}$ appear around $\alpha_{y}$ and $\beta_{y}$ randomly, and there exists the period-doubling bifurcation.

(3) Set $R_{1}$ is composed of the values $\alpha_{y}$ in Eq. (13), one of the endpoints of the maximum invariant interval $E_{y}$ be- 


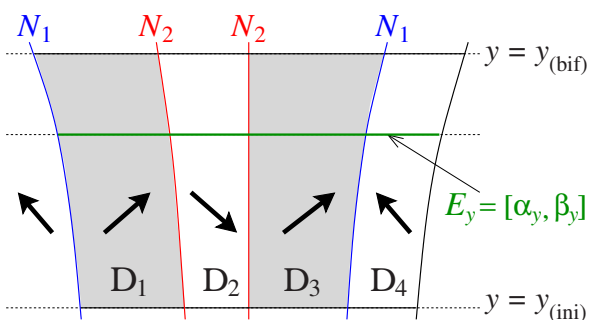

FIG. 3. (Color online) Direction fields of $f$ in $\mathbf{D}_{i}(i=1,2,3,4)$.

cause $\alpha_{y}=r_{y}^{(1)}$ for all $y \in\left[y_{(\mathrm{ini})}, 0\right) \cup\left(0, y^{*}\right]$.

Generally, the period-doubling bifurcation and the tangent bifurcation are generic types of bifurcations, which means that the basic character of the bifurcation cannot be altered by arbitrarily small perturbations that are smooth in the variable and the parameter. That is, if $g_{y}(x)$ is replaced by $g_{y}(x)+\epsilon h(x, y)$, where $h$ is smooth, then, if $\epsilon$ is small enough, the qualitative bifurcation behavior is unchanged. See Ref. 4 for the generic types of bifurcations.

We now consider an orbit of $f, \mathcal{O}_{f}\left(x_{0}, y_{0}\right)$ $=\left\{f^{n}\left(x_{0}, y_{0}\right)\right\}_{n=0}^{\infty}$, where its initial point is $\left(x_{0}, y_{0}\right)=\left(0, y_{(\text {ini) }}\right)$. Under the following assumptions on the sequence $\left(y_{n}\right)$ of $\mathcal{O}_{f}\left(x_{0}, y_{0}\right)$ :

(1) $\left(y_{n}\right)$ keeps moving upward from $y_{\text {(ini) }}$ to $y^{*}$ and

(2) $\left|y_{n+1}-y_{n}\right|$ is sufficiently small,

the tangent bifurcation and the period-doubling bifurcation are suitably embedded in some dynamical orbits of the map $f$ by their generic properties.

In this respect, our goal here is to validate the two assumptions given above for the sequence $\left\{y_{n}\right\}$. This implies that the variable $y$ in Eqs. (2) and (3) plays a role of the bifurcation parameter in $g_{y}$, and so map $f$ follows the dynamics of a random logistic map.

We define a set

$$
\mathbf{D}=\bigcup_{y_{(\text {ini })} \leq y \leq y_{(\text {bif }}}\left\{(x, y) \in \mathbb{R}^{2} \mid \alpha_{y} \leq x \leq \beta_{y}\right\},
$$

and also define four subregions in $\mathbf{D}$ as follows:

$$
\begin{aligned}
& \mathbf{D}_{1}=\left\{(x, y) \in \mathbf{D} \mid f_{1}(x, y)>x, f_{2}(x, y)>y, x<0\right\}, \\
& \mathbf{D}_{2}=\left\{(x, y) \in \mathbf{D} \mid f_{1}(x, y)>x, f_{2}(x, y)<y, x<0\right\}, \\
& \mathbf{D}_{3}=\left\{(x, y) \in \mathbf{D} \mid f_{1}(x, y)>x, f_{2}(x, y)>y, x>0\right\}, \\
& \mathbf{D}_{4}=\left\{(x, y) \in \mathbf{D} \mid f_{1}(x, y)<x, f_{2}(x, y)>y, x>0\right\} .
\end{aligned}
$$

Figure 3 illustrates the placement and appearance of $\mathbf{D}_{i}$ $(i=1,2,3,4)$ and the direction fields of trajectories of $f$ in $\mathbf{D}_{i}$. Due to the property $f_{2}(x, y)<y$ of $\mathbf{D}_{2}$ in Eq. (17), it cannot be guaranteed that the orbit $\mathcal{O}_{f}\left(x_{0}, y_{0}\right)$ keeps moving vertically upward while it travels inside $\mathbf{D}$. We resolve this problem in the following way. For all $(x, y) \in \mathbf{D}_{2}$,

$$
-1<\frac{f_{2}(x, y)-y}{f_{1}(x, y)-x}=\frac{b x-\gamma x^{2} y}{(a-\gamma x)-\left(b x-\gamma x^{2} y\right)}<0
$$

because $(a-\gamma x)>0$ and $\left(b x-\gamma x^{2} y\right)<0$. The boundedness of Eq. (18) yields a constant

$$
\sigma_{\min }=\inf _{(x, y) \in \mathbf{D}_{3}}\left(\frac{f_{2}(x, y)-y}{f_{1}(x, y)-x}\right)
$$

where $-1<\sigma_{\min }<0$. We pick an arbitrary point $\left(x_{n}, y_{n}\right)$ in the orbit that belongs to $\mathbf{D}$.

If $\left(x_{n}, y_{n}\right) \in \mathbf{D}_{4}$, then for sufficiently small $\gamma>0$,

$x_{n}<\beta_{y_{(\text {bif })}}=\frac{2 a(4-\gamma-b)}{4-(\gamma+b)^{2}}<2 a<\frac{a}{\gamma} \Rightarrow a-\gamma x_{n}>0$.

Also, from the fact $x_{n+1}-x_{n}<0$, we obtain

$$
\left(b x_{n}-\gamma x_{n}^{2} y_{n}\right)>a-\gamma x_{n} .
$$

Thus, by Eqs. (20) and (21) we have

$$
\frac{y_{n+1}-y_{n}}{x_{n+1}-x_{n}}<\frac{b x_{n}-\gamma x_{n}^{2} y_{n}}{\left(a-\gamma x_{n}\right)-\left(b x_{n}-\gamma x_{n}^{2} y_{n}\right)}<-1<\sigma_{\min } .
$$

The property of $\mathbf{D}_{4}$ in Eq. (17), that is, $x_{n+1}-x_{n}<0$, yields

$$
y_{n+1}-y_{n}>\sigma_{\min }\left(x_{n+1}-x_{n}\right) \text {. }
$$

On the other hand, we consider the case that $\left(x_{n}, y_{n}\right)$ $\in \mathbf{D}_{1} \cup \mathbf{D}_{2} \cup \mathbf{D}_{3}$. Then

(1) $\left(y_{n+1}-y_{n}\right) /\left(x_{n+1}-x_{n}\right)>0>\sigma_{\min }$ holds for $\left(x_{n}, y_{n}\right)$ $\in \mathbf{D}_{1} \cup \mathbf{D}_{3}$ by Eq. (17).

(2) $\left(y_{n+1}-y_{n}\right) /\left(x_{n+1}-x_{n}\right)>\sigma_{\min }$ holds for $\left(x_{n}, y_{n}\right) \in \mathbf{D}_{2}$ by Eq. (19).

The property of $\mathbf{D}_{1} \cup \mathbf{D}_{2} \cup \mathbf{D}_{3}$ in Eq. (17), that is, $x_{n+1}-x_{n}$ $>0$, yields

$$
y_{n+1}-y_{n}>\sigma_{\min }\left(x_{n+1}-x_{n}\right) .
$$

By Eqs. (22) and (23), $f^{n}\left(x_{0}, y_{0}\right)$ becomes more distant from the line with slope $\sigma_{\min }$ passing through $\left(x_{0}, y_{0}\right)$ up to $y=y_{\text {(bif) }}$ as $n$ increases even if it does not always move upward, and finally, the orbit $\mathcal{O}_{f}\left(x_{0}, y_{0}\right)$ reaches the bifurcation point $y=y_{\text {(bif) }}$. Then from $y=y_{\text {(bif) }}$, the orbit moves along the curve $R_{2}$, so it has no intersection with $\mathbf{D}_{2}$, and hence, it moves strictly upward to $y=y^{*}$, i.e., $y_{n+1}>y_{n}$.

Finally, we show the variation $\Delta y_{n+1}=\left(y_{n+1}-y_{n}\right) / \gamma$ is as small as needed. The orbit $\mathcal{O}_{f}\left(x_{0}, y_{0}\right)$ stably approaches the curve $R_{2}$ when it moves upward from $y=y_{\text {(bif) }}$ to $y=y^{*}$. Thus, we estimate $\Delta y_{n+1}$ only, while $\mathcal{O}_{f}\left(x_{0}, y_{0}\right)$ travels from $y=y_{\text {(ini) }}$ to $y=y_{\text {(bif) }}$. For $y \in\left[y_{\text {(ini) }}, y_{(\text {bif })}\right]$,

$$
\Delta y_{n+1}=\frac{y_{n+1}-y_{n}}{\gamma}=-y x_{n}^{2}+\frac{b}{\gamma} x_{n} .
$$

The minimum $\delta_{\min }$ of $\Delta y_{n+1}$ is

$$
\delta_{\min }=\frac{b^{2}}{4 \gamma^{2} y} \quad \text { at } \quad x_{n}=\frac{b}{2 \gamma y} \in\left[\alpha_{y}, \beta_{y}\right] .
$$

The maximum $\delta_{\max }$ of $\Delta y_{n+1}$ is at $x_{n}=\beta_{y}$, 


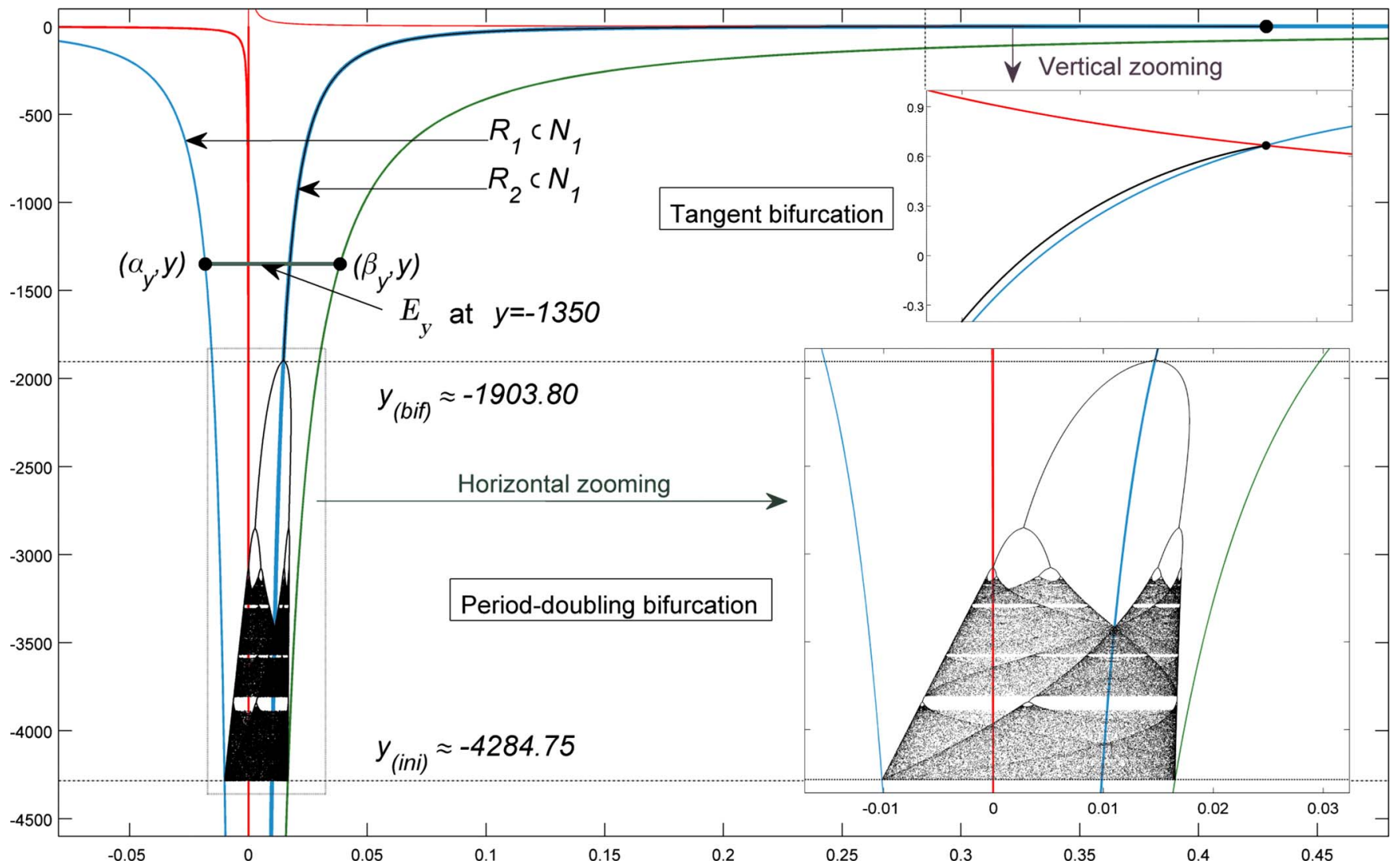

FIG. 4. (Color online) An entire dynamical orbit of $f$ at $a=0.015, b=0.01$, and $\gamma=0.035$. The orbit originates in $(0,-4284.75)$ and converges to the fixed point $(3 / 7,2 / 3)$. It appears around $R_{i}(i=1,2)$, defined in Eq. $(16)$, and it is located between the two boundaries of the invariant interval $E_{y}$ under $g_{y}$ for $y_{(\text {ini })} \leq y$ $\leq y^{*}$. To clearly observe the bifurcation structures in the orbit, we zoom horizontally in the orbit between $y_{(\mathrm{ini})}$ and $y_{(\mathrm{bif})}$ and zoom vertically in the orbit around the fixed point.

$$
\begin{aligned}
\delta_{\max }= & -y \beta_{y}^{2}+\frac{b}{\gamma} \beta_{y} \\
= & \frac{1}{2 \gamma^{2} y}\left((2+\gamma) \sqrt{(\gamma+b)^{2}+4 a \gamma y}\right. \\
& \left.+2-2 \gamma+\gamma^{2}+b \gamma+2 a \gamma y\right) .
\end{aligned}
$$

Thus, $\delta_{\min } \leq \Delta y_{n+1} \leq \delta_{\max }$ holds. For $y_{(\text {ini) }}<y<y_{(\text {bif })}$, the denominator $\gamma^{2} y$ in both $\delta_{\min }$ and $\delta_{\max }$ satisfies

$$
\frac{\gamma}{4 a}\left(4-(\gamma+b)^{2}\right)<\gamma^{2} y<\frac{\gamma}{4 a}\left(9-(\gamma+b)^{2}\right) .
$$

Since $a / \gamma$ and $b / \gamma$ are assumed to be $O(1)$,

$$
\left|\delta_{\min }\right|<c_{1} \text { and }\left|\delta_{\max }\right|<c_{2} \text {, }
$$

where $c_{1}$ and $c_{2}$ are constants and are independent of $\gamma$. Thus, $\Delta y_{n+1}$ is bounded by two fixed constants $\delta_{\min }$ and $\delta_{\max }$ that are independent of $\gamma$. Therefore, $\left(y_{n+1}-y_{n}\right)$ is of the same order as $\gamma$ is, and hence, the sequence $\left(y_{n}\right)$ slowly develops as $n$ increases, as expected.

Figure 4 displays the bifurcation structure embedded in a full dynamical orbit of $f$. It illustrates how the bifurcation structures are associated with the curves of the nullclines $N_{1}$ and $N_{2}$, and the curves in $R_{1}$ and $R_{2}$ defined in Eq. (16). Throughout this figure, we confirm the statements mentioned in Remark 1.

Let us recall

$$
y_{(\text {ini) }}=-\frac{9-(\gamma+b)^{2}}{4 a \gamma}, \quad y_{(\text {bif })}=-\frac{4-(\gamma+b)^{2}}{4 a \gamma} .
$$

Then the smaller $\gamma>0$, the larger the absolute values $\left|y_{\text {(bif) }}\right|$ and $\left|y_{(\text {ini) }}\right|$ and the smaller variation $\Delta y_{n+1}$. Therefore, as $\gamma>0$ decreases, the dynamical orbits of the system possess more evident bifurcation structures. In Sec. V, we shall discuss this fact in more details using numerical results obtained from rescaling of the parameters.

\section{BOUNDED TRAPPING REGIONS}

In this section, we study a bounded trapping region, i.e., a bounded open set $U$ such that $\overline{f(U)} \subset U$. We examine whether a bounded trapping region for the map $f$ exists around the fixed point, and if it exists, we specify a sufficient condition of parameters for the existence of the trapping region. In Sec. III, it was verified that the orbits with bifurcation structures always approach the fixed point, but it has been undetermined whether it converges to the fixed point, it rotates around the fixed point, or even it diverges. The existence of arbitrarily small trapping regions containing the fixed point ensures that some dynamical orbits of $f$ display the bifurcation structures and eventually converge to the fixed point.

The image under $f$ of the vertical line $x=x_{0}$ is a straight line whose equation is 


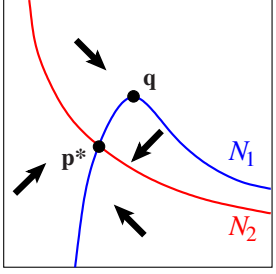

(a) $q>x^{*}$

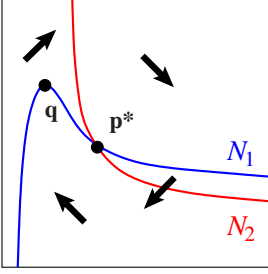

(b) $q \leq x^{*}$
FIG. 5. (Color online) Two possible locations of the maximum point $\mathbf{q}$ of the graph of $N_{1}$ around $\mathbf{p}^{*}$. The arrows represent the direction fields of $f$ around $\mathbf{p}^{*}$ in the subregions divided by $N_{1}$ and $N_{2}$.

$y=\left(-1+\frac{1}{\gamma x_{0}^{2}}\right) x+(1-\gamma) x_{0}+a-\frac{1-\gamma-b}{\gamma x_{0}}-\frac{a}{\gamma x_{0}^{2}}$.

Also, if $f\left(x_{0}, y\right)=f\left(x_{0}, \tilde{y}\right)$, then $y=\tilde{y}$. Thus, when a bounded domain $\mathbf{S}$ is convex in the plane, a sufficient condition for $\mathbf{S}$ to be a trapping region of $f$ is

$$
f(\partial \mathbf{S}) \subset \operatorname{int}(\mathbf{S})
$$

where $\partial \mathbf{S}$ is the boundary of $\mathbf{S}$ and $\operatorname{int}(\mathbf{S})$ is the interior of $\mathbf{S}$.

We build a convex domain containing the fixed point $\mathbf{p}^{*}$ which is bounded in the plane. We set four constants,

$$
\begin{aligned}
& s_{1}=x^{*}-\epsilon_{1} \text { and } s_{2}=x^{*}+\epsilon_{2}, \\
& t_{1}=y^{*}-\delta_{1} \text { and } t_{2}=y^{*}+\delta_{2},
\end{aligned}
$$

where $\epsilon_{i}>0$ and $\delta_{i}>0(i=1,2)$ are as small as needed. Let $\mathbf{S}$ be the rectangular box with four corners,

$$
\mathbf{r}_{1}=\left(s_{1}, t_{1}\right), \quad \mathbf{r}_{2}=\left(s_{2}, t_{1}\right), \quad \mathbf{r}_{3}=\left(s_{2}, t_{2}\right), \quad \mathbf{r}_{4}=\left(s_{1}, t_{2}\right) .
$$

The four sides of $\mathbf{S}$ are denoted by

$$
B_{1}=\overline{\mathbf{r}_{1} \mathbf{r}_{2}}, \quad B_{2}=\overline{\mathbf{r}_{2} \mathbf{r}_{3}}, \quad B_{3}=\overline{\mathbf{r}_{3} \mathbf{r}_{4}}, \quad B_{4}=\overline{\mathbf{r}_{4} \mathbf{r}_{1}} .
$$

We assume that $s_{1}$ and $t_{1}$ is positive so that $\mathbf{S}$ is contained in the first quadrant of the plane. We now find some necessary conditions of parameters for $\mathbf{S}$ to be a trapping region. The graphs of $N_{1}$ and $N_{2}$ in a small neighborhood of $\mathbf{p}^{*}$ are

$$
y=h_{1}(x)=\frac{(\gamma+b) x-a}{\gamma x^{2}}, \quad y=h_{2}(x)=\frac{b}{\gamma x},
$$

respectively. As seen in Fig. 5, the existence of a rectangular trapping region depends on the location of the maximum point $\mathbf{q}=\left(q, h_{1}(q)\right)$ of the graph of $N_{1}$. By examining the direction fields in the subregions divided by $N_{1}$ and $N_{2}$ in a small neighborhood of $\mathbf{p}^{*}$, one can derive a necessary condition for having a rectangular trapping region. If $q \leq x^{*}$, as seen in Fig. 5(b), either no bounded trapping regions exist, or it cannot be rectangular if it exists. Thus, a necessary condition for $\mathbf{S}$ to be a trapping region is that $q>x^{*}$. From the equality $h_{1}^{\prime}(q)=0$, we get the value $q$ as follows:

$$
h_{1}^{\prime}(q)=\frac{-(\gamma+b) q+2 a}{\gamma q^{3}}=0 \Rightarrow q=\frac{2 a}{\gamma+b} .
$$

Then from the inequality $q>x^{*}$, a necessary condition is obtained as follows:

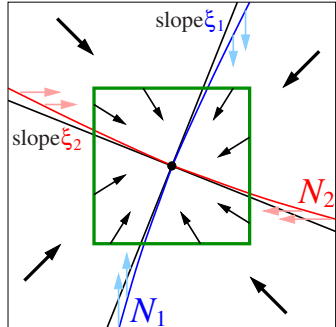

(a) $\left|\xi_{1}\right|>\left|\xi_{2}\right|$

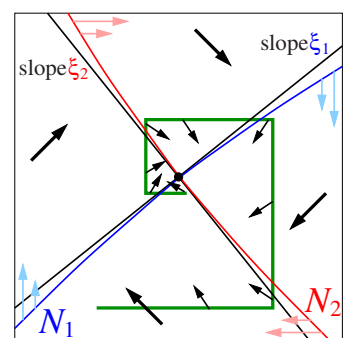

(b) $\left|\xi_{1}\right| \leq\left|\xi_{2}\right|$
FIG. 6. (Color online) Tangent lines of $N_{1}$ and $N_{2}$ around $\mathbf{p}^{*}$. By comparison of the slopes $\xi_{1}$ and $\xi_{2}$ of the two tangent lines, it is examined whether the criterion mentioned in Remark 2 holds.

$$
\frac{2 a}{\gamma+b}>\frac{a}{\gamma} \Rightarrow \gamma>b
$$

We find another necessary condition for $\mathbf{S}$ to be a trapping region. Every point on $N_{1}$ moves vertically by map $f$, while every point on $N_{2}$ moves horizontally. Based on this fact, we have a criterion of the existence of a rectangular trapping region.

Remark 2: The set $\mathbf{S}$ is a trapping region only if the horizontal boundaries $B_{1}$ and $B_{3}$ must intersect $N_{1}$ but not $N_{2}$, and also the vertical boundaries $B_{2}$ and $B_{4}$ must intersect $N_{2}$ but not $N_{1}$.

Let $\xi_{1}$ and $\xi_{2}$ be the slopes of the tangent lines of $N_{1}$ and $N_{2}$ at the fixed point $\mathbf{p}^{*}$, respectively. Under the assumption that $\gamma>b>0$, given in Eq. (27), we have

$\xi_{1}=h_{1}^{\prime}\left(x^{*}\right)=\frac{\gamma^{2}}{a^{2}}-\frac{b \gamma}{a^{2}}>0, \quad \xi_{2}=h_{2}^{\prime}\left(x^{*}\right)=-\frac{b \gamma}{a^{2}}<0$,

where $h_{1}$ and $h_{2}$ are given in Eq. (26). As seen in Fig. 6, when $\left|\xi_{1}\right| \leq\left|\xi_{2}\right|$, it is impossible to construct a rectangular trapping region around $\mathbf{p}^{*}$ which satisfies the criterion in $\mathrm{Re}$ mark 2. Therefore, another necessary condition is that $\left|\xi_{1}\right|>\left|\xi_{2}\right|$

$$
\left|\xi_{1}\right|-\left|\xi_{2}\right|=\frac{\gamma^{2}}{a^{2}}-\frac{2 b \gamma}{a^{2}}>0 \Rightarrow \gamma>2 b .
$$

In this section, instead of assuming that $\gamma$ is sufficiently small and that $a$ and $b$ are of the same order of $\gamma$, we shall assume that

$$
\gamma>2 b \text { and } \gamma>a^{2}
$$

to prove that $\mathbf{S}$ is a rectangular trapping region. Indeed, the additional assumption $\gamma>a^{2}$ in Eq. (29) is secured under the original assumptions given in Sec. II. We prove that $f\left(B_{1}\right)$ and $f\left(B_{3}\right)$ are placed between $B_{2}$ and $B_{4}$, and $f\left(B_{2}\right)$ and $f\left(B_{4}\right)$ are placed between $B_{1}$ and $B_{3}$. This implies $f\left(B_{i}\right) \nsubseteq \mathbf{S}$ holds for $i=1,2,3,4$, that is, the condition given in Eq. (25) holds.

We consider partial derivatives of $f_{1}$ and $f_{2}$ at $\mathbf{p}=(x, y)$ $\in \mathbf{S}$

$$
\left(f_{1}\right)_{x}(x, y)=1-\gamma-b+2 \gamma x y, \quad\left(f_{2}\right)_{y}(x, y)=1-\gamma^{2} x .
$$

Since $x \geq s_{1}>0$ and $y \geq t_{1}>0,\left(f_{1}\right)_{x}(x, y)>0$ holds. This implies that as $x$ increases on the horizontal boundaries of $\mathbf{S}$, $f_{1}\left(x, t_{i}\right)(i=1,2)$ monotone increases, and hence, $f\left(B_{1}\right)$ and $f\left(B_{3}\right)$ are located between $B_{2}$ and $B_{4}$. To complete the proof 


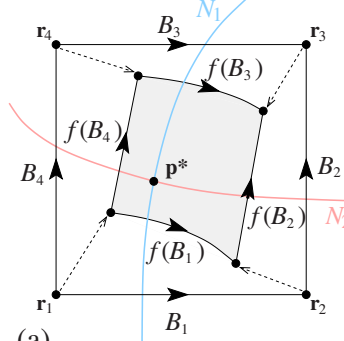

(a)

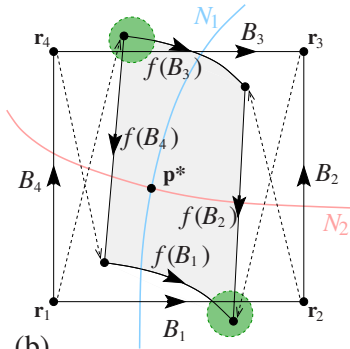

(b)

FIG. 7. (Color online) Two possible cases of the image of $\mathbf{S}$ by $f$. (a) $\left(f_{2}\right)_{y}(x, y) \geq 0$. (b) $\left(f_{2}\right)_{y}(x, y)<0$. In the case of (b), the images $f\left(\mathbf{r}_{1}\right)$ and $f\left(\mathbf{r}_{3}\right)$ must be contained in $\mathbf{S}$.

of Eq. (25), we need to show that $f\left(B_{2}\right)$ and $f\left(B_{4}\right)$ are placed between $B_{1}$ and $B_{3}$.

We suppose $\left(f_{2}\right)_{y}(x, y) \geq 0$. As $y$ increases on the vertical boundaries $B_{2}$ and $B_{4}, f_{2}\left(s_{i}, y\right)(i=1,2)$ monotone increases. Thus, $f\left(B_{2}\right)$ and $f\left(B_{4}\right)$ are located between $B_{1}$ and $B_{3}$, and this completes the statement (25). In this case, the assumption $\gamma>a^{2}$ is not required [see Fig. 7(a)].

On the other hand, we suppose $\left(f_{2}\right)_{y}(x, y)<0$. Since $\left(f_{2}\right)_{x}\left(x^{*}, y^{*}\right)=-b$, there exists an open set $U$ in a small neighborhood of $\mathbf{p}^{*}=\left(x^{*}, y^{*}\right)$ such that $\left(f_{2}\right)_{x}(x, y)<0$ holds for all $(x, y) \in U$. Thus, we have the following:

(1) $f_{2}\left(x, t_{i}\right)(i=1,2)$ monotone decreases as $x$ increases on $B_{1}$ and $B_{3}$ and

(2) $f_{2}\left(s_{1}, t_{i}\right)>f_{2}\left(s_{2}, t_{i}\right)$ for $i=1,2$.

As seen in Fig. 7(b), we need the following inequalities:

$$
f_{2}\left(s_{2}, t_{2}\right)>t_{1} \text { and } f_{2}\left(s_{1}, t_{1}\right)<t_{2} \text {. }
$$

We define an open set $\mathbf{K}$ in the plane by

$$
\mathbf{K}=\left\{(x, y) \in \mathbb{R}^{2}|| \xi_{2}\left|<\frac{\left|y-y^{*}\right|}{\left|x-x^{*}\right|}<\right| \xi_{1} \mid\right\} .
$$

Under the assumption that $\gamma>2 b$ in Eq. (28), $\left|\xi_{2}\right|<\left|\xi_{1}\right|$ holds, so $\mathbf{K}$ is nonempty and it is composed of four connected components in the plane, whose closures intersect only at $\mathbf{p}^{*}$ (see Fig. 8). Following the criterion in Remark 2, we dispose each corner of $\mathbf{S}$ in each subregion of $\mathbf{K}$ without

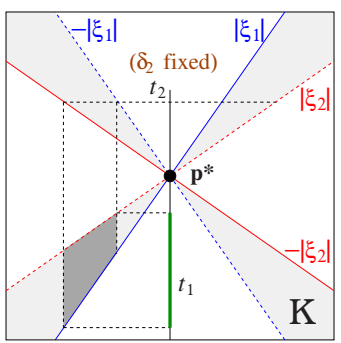

(a)

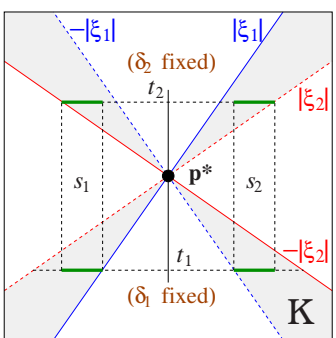

(b)
FIG. 8. (Color online) The region $\mathbf{K}$ (gray color) defined in Eq. (31). Tangent lines (solid lines) of nullclines $N_{1}, N_{2}$ and their symmetric lines (dotted lines) in a neighborhood of the fixed point. Each of the values $\pm\left|\xi_{i}\right|(i=1,2)$ on the lines represents the slope of the line. (a) Location of $t_{1}$. Once $\delta_{2}$ and $t_{2}$ are fixed, $\delta_{1}$ and $t_{1}$ are determined. (b) Locations of $s_{1}$ and $s_{2}$. Once $\delta_{1}, \delta_{2}$ and $t_{1}, t_{2}$ are fixed, $\epsilon_{1}, \epsilon_{2}$ and $s_{1}, s_{2}$ are determined. repetition. Then once $\delta_{2}$ is fixed, $\delta_{1}$ can be determined as follows:

$$
\frac{\left|\xi_{2}\right|}{\left|\xi_{1}\right|} \delta_{2}<\delta_{1}<\frac{\left|\xi_{1}\right|}{\left|\xi_{2}\right|} \delta_{2}
$$

[see Fig. 8(a)]. Also, once $\delta_{1}$ and $\delta_{2}$ are fixed, $\epsilon_{i}(i=1,2)$ must satisfy the following properties:

$$
\frac{\delta_{1}}{\left|\xi_{1}\right|}<\epsilon_{i}<\frac{\delta_{1}}{\left|\xi_{2}\right|} \Rightarrow \frac{a^{2}}{\gamma(\gamma-b)} \delta_{1}<\epsilon_{i}<\frac{a^{2}}{b \gamma} \delta_{1}
$$

and, similarly,

$$
\frac{\delta_{2}}{\left|\xi_{1}\right|}<\epsilon_{i}<\frac{\delta_{2}}{\left|\xi_{2}\right|} \Rightarrow \frac{a^{2}}{\gamma(\gamma-b)} \delta_{2}<\epsilon_{i}<\frac{a^{2}}{b \gamma} \delta_{2}
$$

[see Fig. 8(b)]. Note that if $\delta_{2}$ is sufficiently small, then so are $\epsilon_{1}, \epsilon_{2}$, and $\delta_{1}$. As seen in Eqs. (32)-(34), these constants $\epsilon_{i}$ and $\delta_{i}(i=1,2)$ are bounded by a constant multiple of one of the others. Thus, once one of $\epsilon_{1}, \epsilon_{2}, \delta_{1}$, and $\delta_{2}$ is sufficiently small, so are the others.

We estimate two differences $\left(f_{2}\left(s_{2}, t_{2}\right)-t_{1}\right)$ and $\left(t_{2}-f_{2}\left(s_{1}, t_{1}\right)\right)$ to show that the two differences are both positive to see if the conditions in Eq. (30) hold. We consider

$$
\begin{aligned}
f_{2}\left(s_{2}, t_{2}\right)-t_{1}= & t_{2}+b s_{2}-\gamma s_{2}^{2} t_{2}-t_{1} \\
= & \left(y^{*}+\delta_{2}\right)+b\left(x^{*}+\epsilon_{2}\right) \\
& -\gamma\left(x^{*}+\epsilon_{2}\right)^{2}\left(y^{*}+\delta_{2}\right)-\left(y^{*}-\delta_{1}\right) \\
= & \delta_{1}-\left(b+\frac{b \gamma}{a} \epsilon_{2}\right) \epsilon_{2}+\left(1-\frac{a^{2}}{\gamma}-2 a \epsilon_{2}-\gamma \epsilon_{2}^{2}\right) \delta_{2} .
\end{aligned}
$$

Due to the assumption that $\gamma>a^{2}$, for sufficiently small $\epsilon_{2}>0$,

$$
\left(1-\frac{a^{2}}{\gamma}-2 a \epsilon_{2}-\gamma \epsilon_{2}^{2}\right)>0,
$$

and also, from the inequality (33),

$$
\left(b+\frac{b \gamma}{a} \epsilon_{2}\right) \epsilon_{2}<\left(b+\frac{b \gamma}{a} \epsilon_{2}\right) \frac{a^{2}}{b \gamma} \delta_{1}=\left(\frac{a^{2}}{\gamma}+a \epsilon_{2}\right) \delta_{1}<\delta_{1} .
$$

Thus, $f_{2}\left(s_{2}, t_{2}\right)>t_{1}$ holds. On the other hand, we have

$$
\begin{aligned}
t_{2}-f_{2}\left(s_{1}, t_{1}\right)= & t_{2}-t_{1}-b s_{1}+\gamma s_{1}^{2} t_{1} \\
= & \left(y^{*}+\delta_{2}\right)-\left(y^{*}-\delta_{1}\right)-b\left(x^{*}-\epsilon_{1}\right) \\
& +\gamma\left(x^{*}-\epsilon_{1}\right)^{2}\left(y^{*}-\delta_{1}\right) \\
= & \left(1-\frac{a^{2}}{\gamma}+2 a \epsilon_{1}-\gamma \epsilon_{1}^{2}\right) \delta_{1}+\delta_{2}-\left(b-\frac{b \gamma}{a} \epsilon_{1}\right) \epsilon_{1} .
\end{aligned}
$$

Similarly, due to the assumption that $\gamma>a^{2}$, for sufficiently small $\epsilon_{1}>0$,

$$
\left(1-\frac{a^{2}}{\gamma}+2 a \epsilon_{1}-\gamma \epsilon_{1}^{2}\right)>0,
$$

and also, from the inequality (34), 


$$
\begin{aligned}
\left(b-\frac{b \gamma}{a} \epsilon_{1}\right) \epsilon_{1} & \leq\left(b-\frac{b \gamma}{a} \epsilon_{1}\right) \frac{a^{2}}{b \gamma} \delta_{2} \\
& =\left(\frac{a^{2}}{\gamma}-a \epsilon_{1}\right) \delta_{2}<\delta_{2} .
\end{aligned}
$$

Thus, $f_{2}\left(s_{1}, t_{1}\right)<t_{2}$ holds. Therefore, we obtain the following theorem.

Theorem 3: For all parameters $a>0, b>0$, and $\gamma>0$ satisfying $\gamma>2 b$ and $\gamma>a^{2}$, there exists a bounded trapping region of map $f$ containing the fixed point $\mathbf{p}^{*}$.

Once we build a rectangular trapping region $\mathbf{S}$ around $\mathbf{p}^{*}$, one can build an arbitrarily small rectangular region contained in $\mathbf{S}$, which satisfies all the conditions for trapping region mentioned in this section. Thus, we have

$$
\bigcap_{n=0}^{\infty} f^{n}(\mathbf{S})=\left\{\mathbf{p}^{*}\right\}
$$

and hence, the dynamical orbits converge to the fixed point $\mathbf{p}^{*}$ without rotating around $\mathbf{p}^{*}$ when $\gamma>2 b$ and $\gamma>a^{2}$ hold.

In Theorem 3, the assumption $\gamma>2 b$ corresponds to $B$ $<1 / 2$ in the original system (1). Also, $\gamma>a^{2}$ corresponds to $A^{2}<\gamma^{-1}$, which is true for all sufficiently small discretization steps $\gamma$. Thus, we obtain the following corollary from Theorem 3.

Corollary 4: When $B<1 / 2$, all the solutions of the system (1) converge to the equilibrium but have no limit cycle around the fixed point.

\section{NUMERICAL RESULTS}

In Sec. III, we have understood that the tangent bifurcation and the period-doubling bifurcation are properly embedded in some dynamical orbits of $f$, and the orbits approach the fixed point $\mathbf{p}^{*}$ up to $y=y^{*}$. In Sec. IV, it has been shown that under certain conditions of parameters, there exists a rectangular trapping region containing the fixed point $\mathbf{p}^{*}$, for which such dynamical orbits found in Sec. III converge to $\mathbf{p}^{*}$.

In this section, we rescale the parameters to observe how the embedded bifurcation structures and the bounded trapping regions are developed and transformed. As remarked in Sec. II, while the parameters are rescaled by a small scaling factor, the discretized vector field $f$ holds the same qualitative dynamical properties topologically. We provide some numerical examples of our findings and then confirm that their dynamical properties are unchanged while the parameters being rescaled.

For given parameters $a=a_{0}, b=b_{0}$, and $\gamma=\gamma_{0}$, we consider rescaled parameters by a small factor $\kappa<1$ caused by scaling the discretization step $\Delta t$, that is, $a=\kappa a_{0}, b=\kappa b_{0}$, and $\gamma=\kappa \gamma_{0}$. Let $f_{\kappa}$ be the discretized vector field of the system at the rescaled parameters. Suppose $\mathcal{O}_{\kappa}$ is a dynamical orbit of $f_{\kappa}$ with the initial point $\left(x_{\kappa}, y_{\kappa}\right)$, where $\left(x_{\kappa}, y_{\kappa}\right)$ are placed between $R_{1}$ and $R_{2}$, and

$$
y_{\kappa}=-\frac{9-\kappa^{2}\left(\gamma_{0}+b_{0}\right)^{2}}{4 \kappa^{2} a_{0} \gamma_{0}} \text {. }
$$

Here, $y_{\kappa}$ is the value in Eq. (15) at the rescaled parameters, and so $x_{\kappa}$ is contained in the maximal invariant interval $E_{y_{\kappa}}$.
Thus, a bifurcation structure is embedded in $\mathcal{O}_{\kappa}$. The graphs $h_{1}$ and $h_{2}$, given in Eq. (26), are independent of rescaling of parameters, so that for all scaling factor $\kappa \in(0,1]$, we have the same nullclines $N_{1}$ and $N_{2}$ for $f_{\kappa}$ whose equations are

$$
h_{1}(x)=\frac{\left(\gamma_{0}+b_{0}\right) x-a_{0}}{\gamma_{0} x^{2}} \text { and } h_{2}(x)=\frac{b_{0}}{\gamma_{0} x} .
$$

Thus, for each $\kappa \in(0,1]$, the orbit $\mathcal{O}_{\kappa}$ appears around the same nullclines and so does the bifurcation structures. In Fig. 9, we deal with a few sets of rescaled parameters to obtain successive bifurcation structures of dynamical orbits that appear around the same nullclines. It is observed that the dynamical orbits move upward along the same nullclines and converge to the same fixed point.

In this paper, it is assumed that $\gamma$ is sufficiently small and $a$ and $b$ are of the same order of $\gamma$. Indeed, even without these assumptions, a bifurcation structure is embedded in the vector field in the same way as in Sec. III. However, due to the magnitudes of the variations $\Delta x$ and $\Delta y$, it is not so clearly observable until the parameter $\gamma$ is sufficiently small. At the end of Sec. III, it was remarked that the smaller the parameter $\gamma$, the more evident the bifurcation structure embedded in the direction field. In Fig. 10, we confirm that the smaller the scaling factor $\kappa$, the longer, thinner, and more apparent the bifurcation structure in the orbit $\mathcal{O}_{\kappa}$. Moreover, it is observed that the shapes of bifurcation structures for the rescaled parameters are the same as the others.

Finally, we generalize map $f$ as follows. We consider the two-dimensional map $f^{(r)}: \mathbb{R}^{2} \rightarrow \mathbb{R}^{2}$ defined by $f^{(r)}=\left(f_{1}^{(r)}, f_{2}^{(r)}\right)$, where

$$
\begin{aligned}
& f_{1}^{(r)}(x, y)=a+(1-\gamma-b) x-\gamma x^{2} y^{r}, \\
& f_{2}^{(r)}(x, y)=y+b x-\gamma x^{2} y^{r} .
\end{aligned}
$$

Then for all $r>0, f^{(r)}$ possesses the same properties as $f$ does. Map $f^{(r)}$ has a bifurcation structure in its orbit and also there exists a bounded trapping region for some range of parameters. Using the same argument in Secs. III and IV, one can verify these properties for $f^{(r)}$. Figure 11 displays the bifurcation structures in the orbit of $f^{(r)}$ in the cases of $r=0.5$ and $r=2$ in Eq. (36).

A question arises here. How are these bifurcation structures observed in the case of infinitesimal discretization of time step? The original Brusselator cannot produce such complicated orbits because of its differentiability and dimensionality. As long as the scaling factor is nonzero, there is a bifurcation structure in the orbit of $f$ that produces an oscillation around a nullcline. As the scaling factor becomes smaller, the bifurcation structure gets longer and thinner and, hence, the amplitude of the oscillation of the orbit gets smaller. Suppose that the scaling factor is infinitesimal. Then the orbit forms a flow representing a solution of the original system. However, since the scaling factor is nonzero, the oscillation of the orbit is still alive but infinitesimal. Thus, while the flow converges to the equilibrium, it must experience an oscillation, which is invisible.

More specifically, we consider the following. As the scaling factor $\kappa>0$ becomes infinitesimal, the orbit $\mathcal{O}_{\kappa}$ turns 


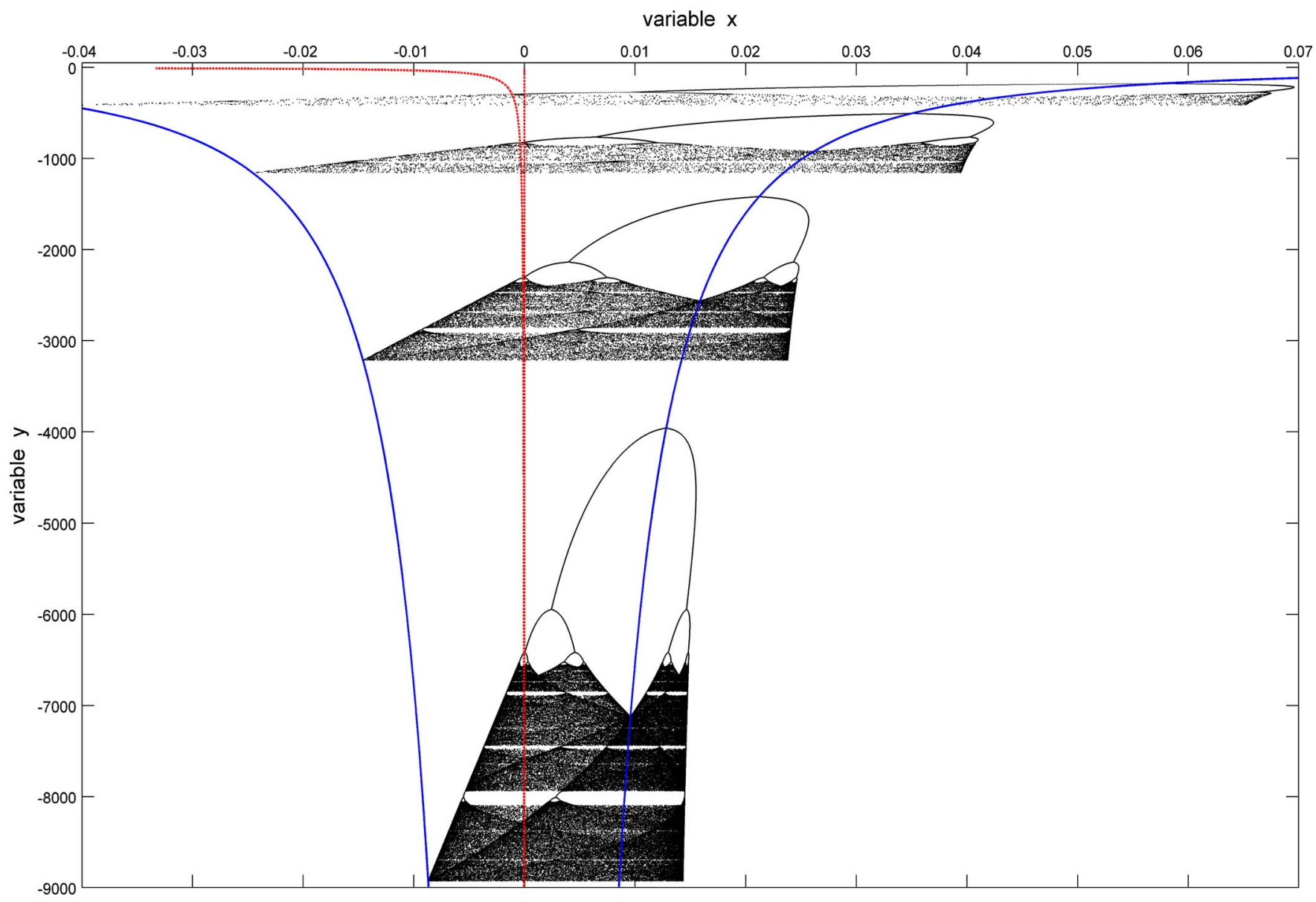

FIG. 9. (Color online) Bifurcated evolutions obtained by rescaling the parameters. The two outer curves are of $N_{1}$, while the inner curves are of $N_{2}$. The topmost bifurcation structure is obtained from the parameters $a=0.06, b=0.03$, and $\gamma=0.09$. The successive each bifurcated evolution is rescaled by the factor of 0.6 from the respective upper one.

out to be a flow $\phi^{t}=\phi^{t}(x, y)$ that is a solution of the system of ODEs in Eq. (1). Since the system (1) has the same nullclines $N_{1}$ and $N_{2}$ as in Eq. (35), the initial point $\left(x_{\kappa}, y_{\kappa}\right)$ of $\phi^{t}$ is still located between the $y$-axis and the curve $R_{2}$, and $\left|y_{\kappa}\right|$ becomes arbitrarily large. The flow $\phi^{t}$ intersects all the maximal invariant intervals $E_{y}$ for all $y \in\left[y_{\kappa}, y^{*}\right]$ because all the mathematical expressions in Sec. III hold for all sufficiently small $\gamma>0$. Thus, by Corollary 4 , under the assumption that $B<1 / 2$, the flow $\phi^{t}$ moves upward along the curves $R_{2} \subset N_{1}$ and eventually converges to the equilibrium $\mathbf{p}^{*}$ as the orbit $\mathcal{O}_{\kappa}$ do so. However, the flow $\phi^{t}$ cannot cross the curve, and it is just placed next above $R_{2}$. This means that $\phi^{t}$

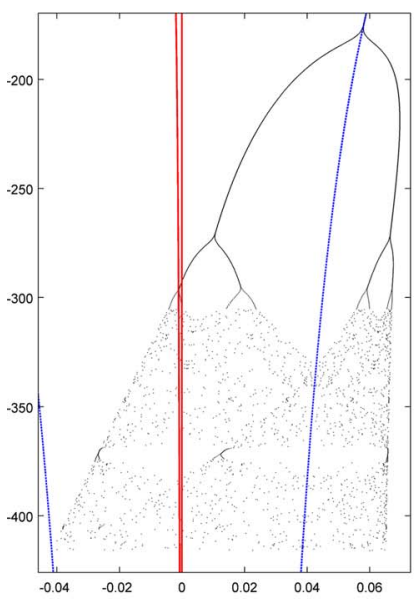

(a) Parameters given at first

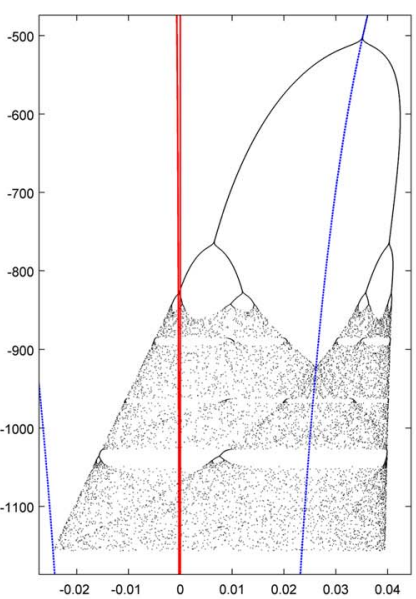

(b) those rescaled by 0.6

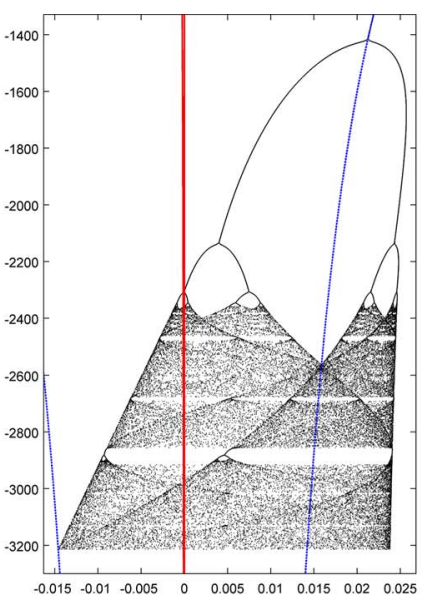

(c) those rescaled by $0.6^{2}$

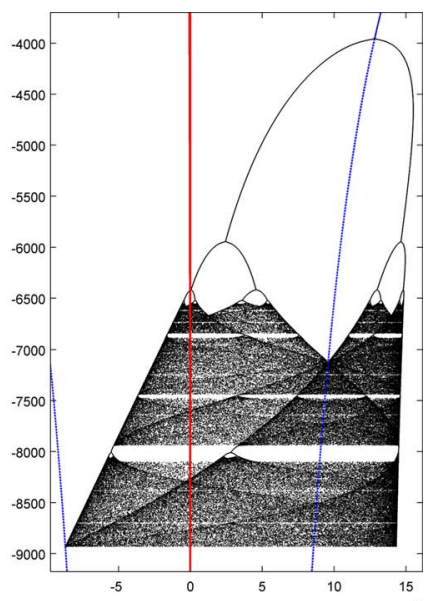

(d) those rescaled by $0.6^{3}$

FIG. 10. (Color online) Bifurcated evolution rescalings. (a)-(d) correspond to the bifurcation structures in Fig. 9 from the top to the bottom. 

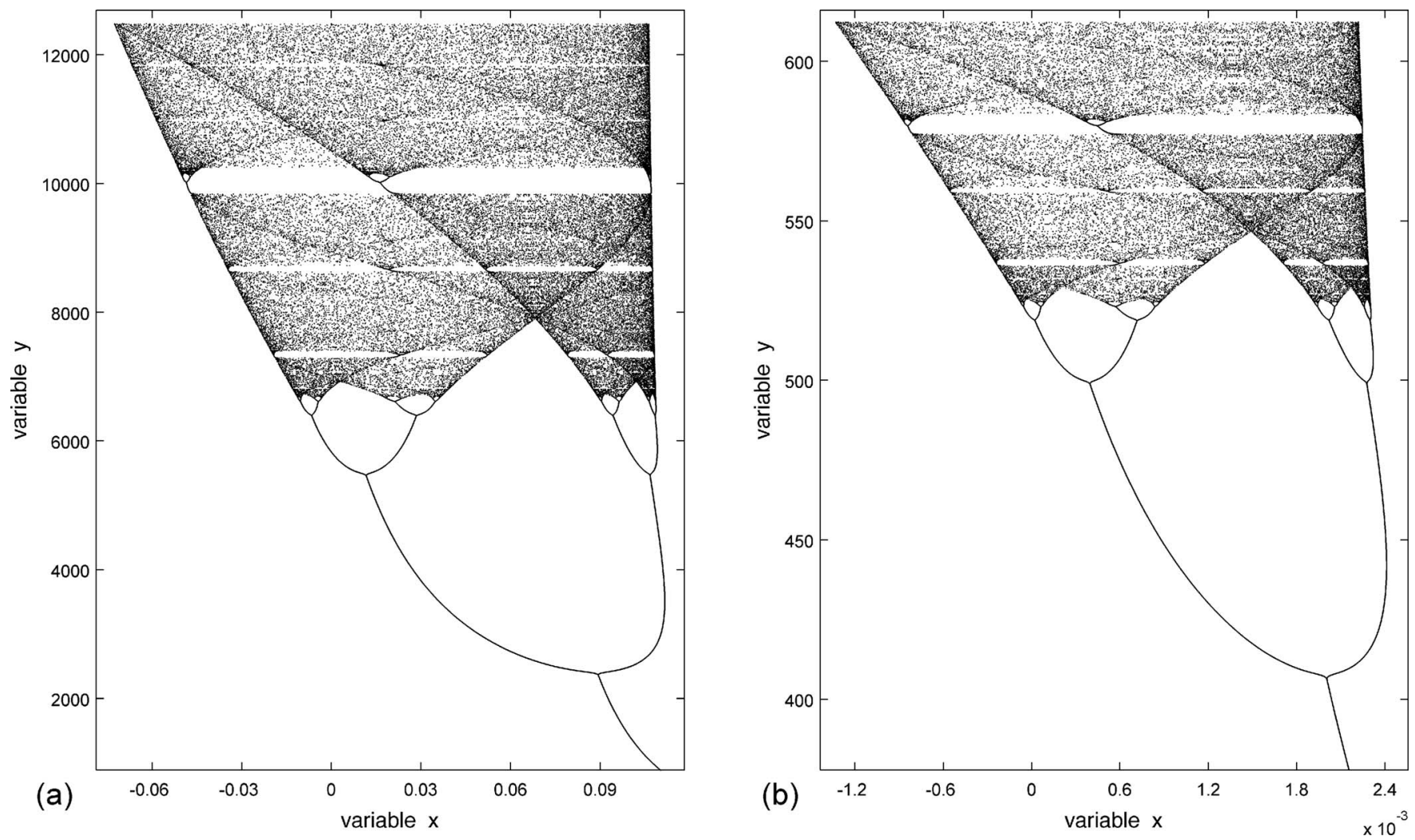

FIG. 11. Bifurcation structures in a dynamical orbit of $f^{(r)}$ defined in Eq. (36). (a) The orbit originating in $(012481.08)$ at $a=0.1, b=0.05, \gamma=0.2$, and $r$ $=0.5$. (b) The orbit originating in $(0,612.37)$ at $a=0.002, b=0.001, \gamma=0.003$, and $r=2$.

smoothly evolves along the line $R_{2}$ with no oscillating behaviors, while the orbit $\mathcal{O}_{\kappa}$ exhibits the bifurcation structure around $R_{2}$.

The essence of generating the embedded bifurcation structures is that the orbit $\mathcal{O}_{\kappa}$ crosses the curve $R_{2}$ sufficiently many times. However, the flow $\phi^{t}$ of the original Brusselator cannot do so. Thus, by adding small perturbation terms to the original system, we may have $\phi^{t}$ cross $R_{2}$ sufficiently many times while keeping most of properties of the original Brusselator. The ways to give the perturbation terms to the original Brusselator are (1) to add spatial diffusion terms to the system to produce the reaction-diffusion equa-

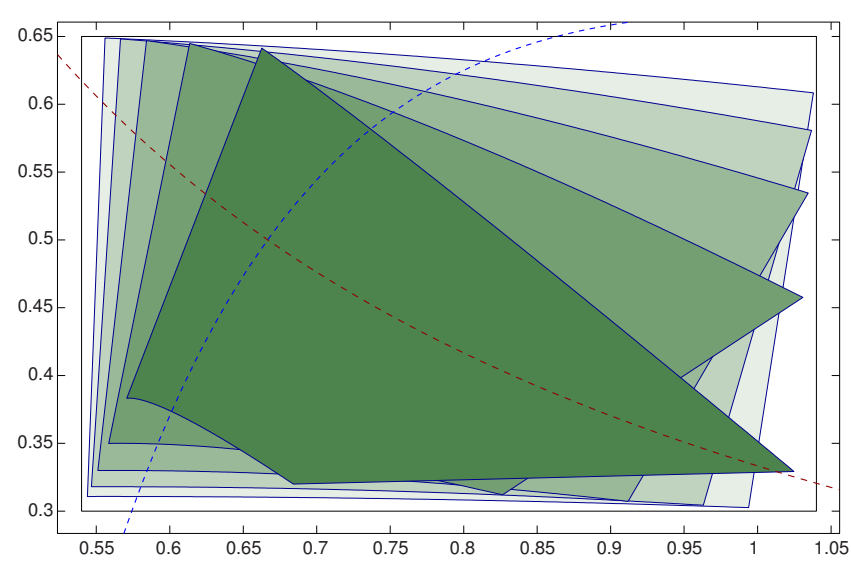

FIG. 12. (Color online) A trapping region $\mathbf{S}=[0.54,1.04] \times[0.3,0.65]$ and its images under $f$ at the parameter values $a=0.6 \kappa, b=0.3 \kappa$, and $\gamma=0.9 \kappa$ for $\kappa=0.6^{n}, n=1,2,3,4,5$ from front to back in order of the images. tion and (2) to add a periodic term or a stochastic term to the system to make a periodic forced Brusselator or a stochastic forced Brusselator (see Ref. 5).

Suppose that $\gamma_{0}>2 b_{0}$ and $\gamma_{0}>a_{0}^{2}$. Then by Theorem 3, there is a rectangular trapping region $\mathbf{S}$ for $f_{\kappa}$ when $\kappa=1$. For all $\kappa \in(0,1)$, we have

$$
\kappa \gamma_{0}>2 \kappa b_{0} \text { and } \kappa \gamma_{0}>\left(\kappa a_{0}\right)^{2} .
$$

Thus, there also exist bounded trapping regions for $f_{\kappa}$. Actually, one can confirm that all the mathematical expressions in Sec. IV are true for all rescaled parameters by $\kappa$. Thus, $\mathbf{S}$ is a rectangular trapping region for $f_{\kappa}$ for all $\kappa \in(0,1)$. This is the reason that the original system possesses the same trapping region as the discretized vector field (see Fig. 12).

It is a well-known fact that the original Brusselator (1) has a trapping region for all values of the system parameters $A>0$ and $B>0$ (see Refs. 6 and 7). Thus, the solution of the system converges to either a limit cycle or the equilibrium, depending on the stability of the equilibrium.

\section{CONCLUSION}

In this paper, we studied dynamical behaviors of some orbits in the discretized vector fields of the Brusselator. Bifurcation structures are embedded in some orbits in the direction field. The orbits enter to stay in bounded trapping regions containing the fixed point and eventually converge to the fixed point. Additionally, we rescaled the parameters to 
observe how the dynamical properties of orbits concerning the embedded bifurcation structures and the bounded trapping regions are evolved.

Furthermore, it would be interesting to note the similarity of the present bifurcation phenomena to biological evolution. A slowly varying $y$ variable may be viewed as genes, and a relatively fast varying $x$ variable as representing phenotypic expressions. Biological evolution is said to be a process of increasing diversity in all species, but it can also be viewed as an opposite process, restricted to one species because of the presence of the selection mechanisms. The selection mechanisms through adaptation processes to the environments can reduce the complexity of the behaviors. Then, it is not surprising to see characteristics of biological evolution in the dynamics of evolution equations.

\section{ACKNOWLEDGMENTS}

The authors would like to express their special thanks to Ya. Pesin and M. Yuri for continual encouragements, to $\mathrm{K}$. Zhang for critical proofreading, and to M. Maeda for double- checking scientific numerical computations. This work was partially supported by Grant-in-Aid for Scientific Research on Priority Areas "Integrative Brain Research" (Grant No. 18019002), partially supported by Grant-in-Aid for Scientific Research on Priority Areas "Understanding of Mobiligence" (Grant No. 18047001), partially supported by Grant-in-Aid for Scientific Research (B) (Grant No. 18340021), and partially supported by Grant-in-Aid for Exploratory Research (Grant No. 17650056) — all from the Ministry of Education, Culture, Sports, Science and Technology of Japan.

${ }^{1}$ I. Prigogine and R. Lefever, J. Chem. Phys. 48, 1695 (1968).

${ }^{2}$ H. Kang, Discrete Contin. Dyn. Syst. 20, 939 (2008).

${ }^{3}$ H. Kang and Ya. B. Pesin, Milan J. Math 73, 1 (2005).

${ }^{4}$ E. Ott, Chaos in Dynamical Systems, 2nd ed. (Cambridge University Press, Cambridge, 2002).

${ }^{5}$ I. Bashkirtseva and L. Ryashko, Chaos, Solitons Fractals 26, 1437 (2005).

${ }^{6} \mathrm{~J}$. Guckenhheimer and P. Holmes, Nonlinear Oscillations, Dynamical Systems, and Bifurcations of Vector Fields (Springer, New York, 1983).

${ }^{7}$ S. H. Strogatz, Nonlinear Dynamics and Chaos (Westview Press, Cambridge, Massachusetts, 1994). 